\title{
Menstrual hygiene among adolescent school students: An in- depth cross-sectional study in an urban community of WestBengal, India
}

\author{
Dr Shamima Yasmin ${ }^{1,}$ Dr Nirmalya Manna ${ }^{2,}$ Dr Sarmila Mallik ${ }^{3,}$ \\ Dr Ashfaque Ahmed ${ }^{4}$ Dr Baisakhi Paria ${ }^{5}$ \\ ${ }^{I}$ Department of Community Medicine,NRS Medical College, Kolkata, India \\ ${ }^{2}$ Department of Community Medicine Medical College, Kolkata, India \\ ${ }^{3}$ Department of Community Medicine, Murshidabad Medical College, India \\ ${ }^{4}$ Department of Medicine Medinipur Medical College, India \\ ${ }^{5}$ Department of Community Medicine, Calcutta National Medical College, India
}

\begin{abstract}
Menstrual hygiene is an issue that is insufficiently acknowledged. Menstruation and menstrual practices are still clouded by taboos and socio-cultural restrictions resulting in adolescent girls remaining ignorant of the scientific facts and hygienic health practices, which sometimes result into adverse health outcomes. The present study was designed to assess the knowledge, beliefs, and source of information regarding menstruation among the adolescent school girls of the secondary school and also to identify the status of menstrual hygiene among them. A descriptive, cross-sectional community based study was conducted among 147 adolescent girls of a secondary school situated in the urban field practice area of dept of Community Medicine Medical College Kolkata with the help of a pre-designed and pre-tested questionnaire. Out of 147 respondents, $62(42 \%)$ girls were aware about menstruation prior to attainment of menarche. Hand-washing was regular among $91.8 \%$ but $16.3 \%$ washed only with water. Similarly washing of private parts were regular among $76.9 \%$ but $74.1 \%$ used only water no soap, there is significant relationship between hygienic practices followed and presence of continuous supply of water and presence of exclusive toilet of their family. Except for $2(1.3 \%)$ everybody followed some taboo or unnecessary restriction. Menstrual hygiene, a very important risk factor for reproductive tract infections (RTI), is a vital aspect of health education. This problem, unfortunately, has not yet been addressed seriously in terms of its mental, social and health impact on the country.
\end{abstract}

Keywords: Adolescent girl, menstrual hygiene, RTI, sanitary pad

\section{Introduction}

Menstrual hygiene is an issue that is insufficiently acknowledged and has not received adequate attention in the reproductive health and Water, Sanitation and Hygiene (WASH) sectors in developing countries including India and its relationship with and impact on achieving many Millennium Development Goals (MDGs) is rarely acknowledged. Studies that make the issue visible to the concerned policymakers and inform practical actions are very much warranted. Menstruation is a phenomenon unique to the females. ${ }^{1}$ The first menstruation (menarche) occurs between 11 and 15 years with a mean of 13 years. ${ }^{2}$ Menstruation is still regarded as something unclean or dirty in Indian society. The reaction to menstruation depends upon awareness and knowledge about the subject. The manner in which a girl learns about menstruation and its associated changes may have an impact on her response to the event of menarche. ${ }^{3}$ Although menstruation is a natural process; it is linked with several misconceptions and practices, which sometimes result into adverse health outcomes. Hygiene related practices of women during menstruation are of considerable importance, especially in terms of increased vulnerability to reproductive tract infections (RTI). ${ }^{4}$ The interplay of socio-economic status, menstrual hygiene practices and RTI are noticeable. Women having better knowledge regarding menstrual hygiene and safe practices are less vulnerable to RTI and its consequences. ${ }^{5}$ Therefore, increased knowledge about menstruation right from childhood may escalate safe practices and may help in mitigating the suffering of millions of women. With this background the present study was undertaken to assess the knowledge, beliefs, and source of information regarding menstruation among the adolescent school girls of the secondary school and also to identify the status of menstrual hygiene among them.

\section{Methodology}

It was an observational, descriptive community based study with cross-sectional design among adolescent school students. The study was conducted in Victoria Institution from October to December 2010. Out of the total 8 schools situated in the urban field practice area of the Department of Community Medicine, Medical College, Kolkata this school was selected by simple random sampling method. 
Adolescent girls who had attained menarche were only included in the study and constituted the study population. Adolescents who were present in the schools during the days of survey constituted the sample size. From the previous studies it was revealed that mean age of menarche was 13.2 years. ${ }^{5}$ Hence students of only class IX onwards was decided to be included in the study. The study was approved by the Institutional Ethics Committee of Medical College Kolkata. A pre-designed pre-tested semi-structured questionnaire was prepared for collection of data. The school authority was contacted and the purpose of the study was explained. After obtaining the permission from the school authorities, the school was visited as per pre-planned schedule for getting information from the adolescent girls. Mutually suitable days of the week were fixed when the students had games classes or other non-academic classes. A list of adolescent girls who had attained menarche was prepared beforehand with the help of the school teachers. On the appointed day the eligible students were gathered in a hall and were made to sit at a fair distance from each other to avoid peeping and discussion. The adolescent girls were explained about the purpose of the study and were assured of confidentiality. A verbal consent was obtained from the girls before administering the questionnaire which included questions regarding the knowledge and awareness about menstruation, the source of information and practices followed to maintain menstrual hygiene. They were instructed on how to fill the questionnaire. Adequate time was given to fill up the questionnaire. After completing the questionnaire, the students were asked to place their sheets in a box provided at the corner of the hall. This was followed by a session educating the girls about the normal physiology of menstruation, the importance of maintaining hygiene and safe hygienic practices during menstruation. Questions and concerns of the participants were also addressed at the end of the session. No follow-up visit was planned to track the absentees due to shortage of time and also to maintain the confidentiality of the questionnaire and truthfulness of the responses. Data regarding menstrual hygiene were collected from 147 adolescent girls. Data were then compiled in an Excel sheet and were analyzed by using Epi Info $^{\mathrm{TM}}$ Version 3.5.1 (Release Date: August 18, 2008). Relevant statistical tests were applied to study the test of significance.

\section{Results}

The study showed that age of the respondents $(\mathrm{n}=147)$ varied from $13-18$ years. The students of class IX were mostly $14-15$ years (36\%) and students of class XI mostly 16-17 years (60\%) with mean age 15.5 years and standard deviation 1.18 years.

The study population were mainly Hindu (72.1\%). and nuclear family $(60 \%)$, number of family member being between 5-10, the education of their mothers are mostly madhyamik (class X Pass - 39.5\%), below madhyamik was $11.6 \%$, higher-secondary was $19.7 \%$, graduate and above was $29.2 \%$. We tried to assess the socio-economic status but non-response was for $95(64.6 \%)$ students, the school students were unable to tell their family income. Out of the students who have answered $(n=52)$, most of them $(30.7 \%)$ were in class II of Modified Prasad scale. ${ }^{6}$

Mean age of menarche was 11.95 years with a SD of 1.08, range between 8-14 years. $42 \%$ knew about menstrual cycle before their menarche. In most cases their first informant was their friend. Only $63.3 \%$ knew bleeding occurs from uterus, $72.8 \%$ knew it to be a physiological process (table 1).

$82.3 \%$ used sanitary pad only. 23(15.7\%) of the respondents uses old washed cloth and out of them $16(69.5 \%)$ had problem while washing and drying of the cloth which mostly consisted of lack of privacy (93.7\%). keeping the cloth in places away from prying eyes became a problem for them and for that they usually dried and stored in un-hygienic places. 98.6\% of the respondents followed some restriction or taboo during menstruation (table 2). Regarding hygienic practices during menstruation $85.7 \%$ had daily bath. Others felt bathing should be restricted in the first two days of menstruation as bathing increases the menstrual flow. Handwashing with soap and water was present in $83.7 \%$, and cleaning of external genetalia with soap and water was present only in $25.9 \%$ of respondents (table 3 ). It was seen that religious taboos and restriction on type of food was maximum in Muslims (97.3\% and 59.4\% respectively). Such restrictions were also present in respondents whose mother's education was above graduation (table 4). $80.3 \%$ of the respondents had toilet exclusive to the family but $19.7 \%$ uses common toilet with user per toilet being mostly $15-20$ persons. $38.8 \%$ of respondents' family had to keep water stored in their toilet. $10.2 \%$ of respondents complained of excessive white discharge. It was seen that hygienic practice like hand washing, daily bath had significant relationship with presence of continuous supply of water and presence of toilet exclusive to their family (table 5).

\section{Discussion}

In the present study, the mean age of menarche of the respondents was 11.95 years, whereas in a study conducted in Rajasthan in 2005 by Khanna et al ${ }^{7}$ the mean age at menarche was found to be 13.2 years. Unfortunately, 58\% girls were ignorant about menstruation before menarche in this study. But, each and every girl child should be aware about menstruation, ideally a mother should be the main informant at this tender age. In a study conducted among 664 schoolgirls aged 14-18 in Mansoura, Egypt by El-Gilany et al. ${ }^{8}$ mass media 
were the main source of information about menstrual hygiene, followed by mothers. Another study conducted by Deo et al. ${ }^{9}$ reported that $40(42.5 \%)$ urban and $41(55.4 \%)$ rural girls were aware about menstruation prior to attainment of menarche. In urban girls, mother was the main source of information about menstruation (27.5\%), whereas it was teacher in the rural counterparts $(27.01 \%)$. Other sources of information were friends, relatives and books.

It was observed in this study that $72.8 \%$ girls believed it to be a physiological process, whereas in a similar study conducted in Rajasthan by Khanna et al. ${ }^{7}$, nearly $70 \%$ believed that menstruation was not a natural process. Similar research done in Andhra Pradesh University by Drakshayani et a $1^{10}$ detected that around $78.5 \%$ knew menstrual bleeding originated from the uterus. In this study it was $63.3 \%$

A study by Dasgupta et $\mathrm{al}^{11}$ in a rural community showed that majority of the girls preferred cloth pieces rather than sanitary pads as menstrual absorbent. Only $11.25 \%$ girls used sanitary pads during menstruation. In a study conducted in Rajasthan by Khanna et $\mathrm{al}^{7}$, three-fourths of the girls used old cloth during their periods and only one-fifth reported using readymade sanitary pads. It was observed that the usual practice was to wash the cloth with soap after use and keep it at some secret place till the next menstrual period. To maintain privacy these are sometimes hidden in unhygienic places. In this study although only 23 (15.7\%) used old washed cloth $30 \%$ faced problem of lack of place, water, privacy while washing and drying cloth pieces Health education should be developed to empower young women with sufficient knowledge so that they shift to appropriate health-taking behaviors. Educating young girls about the routine practice of taking a bath with warm water in the early days of menstrual period, would not only lead to the development of positive mental and social behaviors, but could also be effective in reducing hygiene problems in the community

Considering the limitations of this study in terms of sampling method, additional studies may be needed using a wider geographic scope and a larger sample size that should include young girls and their mothers, are proposed in order to produce sufficient and comprehensive results

\section{Conclusion}

This study was conducted to ascertain menstrual hygiene among adolescent girls. The study revealed that menstrual hygiene was far from satisfactory among a large proportion of the adolescents. Lack of appropriate and sufficient information about menstrual hygiene can be attributed to cultural and religious beliefs and taboos. Thus, the above findings reinforce the need to encourage safe and hygienic practices among the adolescent girls and bring them out of traditional beliefs, misconceptions and restrictions regarding menstruation. General awareness about cause, organs involved in menstruation was to be improved. Use of sanitary napkins was to be enhanced by social marketing. For using old washed cloth, proper hygiene was to be maintained. Incorrect restrictions, myths and beliefs associated with menstruation can be removed by the help of teachers and parents.

Table 1: Distribution of respondents according to their Knowledge about menstruation $(\mathrm{n}=147)$

\begin{tabular}{|l|c|c|}
\hline Attributes & Number & Percentage \\
\hline Knew about menstruation Before menarche & 62 & 42 \\
Yes & 85 & 58 \\
No & 27 & 18.3 \\
\hline Source of knowledge of menstrual cycle before menarche & 5 & 3.4 \\
Mother & 30 & 20.4 \\
Relative & 85 & 57.9 \\
Friend & & \\
Did not know & 93 & 63.3 \\
\hline Knowledge of organ from where bleeding occurs & 10 & 6.8 \\
Uterus & 44 & 29.9 \\
Bladder & & \\
Do not know & 107 & 72.8 \\
\hline Knowledge of cause of menstruation & 15 & 10.2 \\
Physiological & 25 & 17.0 \\
God given & & \\
Do not know & & \\
\hline
\end{tabular}


Table 2: Distribution of respondents according to their practice during menstruation

\begin{tabular}{|l|c|c|}
\hline Attributes & Number & Percentage \\
\hline Type of absorbent $(\mathrm{n}=147)$ & & 82.2 \\
Sanitary pads & 21 & 1.4 \\
New cloth & 22 & 15.0 \\
Old washed cloth & 1 & 0.7 \\
Pad + old washed cloth & 1 & 0.7 \\
Pad + new cloth & & \\
\hline Problems faced while using old washed cloth during washing & & 70 \\
and drying (n=23) & 16 & 30 \\
Yes & 7 & \\
No & & 50 \\
\hline Type of problem faced while using old washed cloth during & & 62.5 \\
washing and drying (n=16)** & 8 & 93.75 \\
Shortage of water & 10 & \\
Scarcity of place & 15 & 9.52 \\
Lack of privacy & & 90.47 \\
\hline Different restriction among adolescent girls during & & \\
menstruation (n=145)** & 133 & 71 \\
Religious place & 27 & 14 \\
Food & & \\
Games & & \\
Marriage & & \\
\hline
\end{tabular}

** (multiple responses)

Table 3: Distribution of respondents according to their hygienic practices during menstruation

\begin{tabular}{|l|c|c|}
\hline Hygienic practices during menstruation & Number & Percentage \\
\hline Daily bath & 126 & \\
Yes & 21 & 85.7 \\
No & & 14.3 \\
\hline Hand Washing & 135 & 91.8 \\
Regular* & 12 & 8.2 \\
Irregular & 24 & \\
\hline Hand Washing with & 123 & 16.3 \\
Water & & 83.7 \\
Soap and water & 113 & \\
\hline Cleaning of external genitalia & 34 & 76.9 \\
Regular** & & 23.1 \\
Irregular & 109 & \\
& 38 & 74.1 \\
\hline Cleaning of external Genitalia with & & 25.9 \\
\hline Water & & \\
Soap and water & & \\
\hline
\end{tabular}

* Regular: hand washing every time after visit to the toilet during menstruation;

$* *$ Regular: Frequency of cleaning of external genitalia is $\geq 2$ day; 
Table 4: Distribution of respondents according to the restriction followed during menstruation with their mother's education and their religion

\begin{tabular}{|l|c|c|}
\hline Attributes & $\begin{array}{c}\text { Religious taboo } \\
\text { present }\end{array}$ & $\begin{array}{c}\text { Restriction of food } \\
\text { present }\end{array}$ \\
\hline Religion & & \\
Hindu $(\mathrm{n}=106)$ & $96(90.5 \%)$ & $48(45.2 \%)$ \\
Muslim (n=37) & $36(97.3 \%)$ & $22(59.4 \%)$ \\
Christian (n=4) & $1(25 \%)$ & $1(25 \%)$ \\
\hline Education of mother & $16(94.1 \%)$ & $10(58.8 \%)$ \\
Below Madhyamik (n=17) & $51(87.9 \%)$ & $29(50 \%)$ \\
Madhyamik (n=58) & $26(89.6 \%)$ & $14(48.2 \%)$ \\
Higher Secondary $(\mathrm{n}=29)$ & $40(93 \%)$ & $18(41.8 \%)$ \\
Graduate \& above $(\mathrm{n}=43)$ & & \\
\hline
\end{tabular}

Table 5: Distribution of respondents according to their hygienic practices during menstruation along with presence of stored water and exclusive toilet for their family

\begin{tabular}{|l|c|c|c|}
\hline \multicolumn{1}{|c|}{ Attributes } & Regular Hand washing & $\begin{array}{c}\text { Regular Cleaning of } \\
\text { private parts }\end{array}$ & Daily bath \\
\hline Stored Water & $47(82.5 \%)$ & $41(71.9 \%)$ & $41(71.9 \%)$ \\
Yes (n=57) & $88(97.8 \%)$ & $72(80 \%)$ & $85(94.4 \%)$ \\
No (n=90) & 8.98 & 1.28 & 12.68 \\
$\chi^{2}$ & 1 & 1 & 1 \\
df & $<0.001$ & 0.25 & $<0.001$ \\
p Value & $117(99.2 \%)$ & $98(83.1 \%)$ & $113(95.8 \%)$ \\
\hline Separate Bathroom & $18(62.1 \%)$ & $15(51.7 \%)$ & $13(44.8 \%)$ \\
Yes (n=118) & 37.9 & 11.15 & 45.25 \\
No (n=29) & 1 & 1 & 1 \\
$\chi^{2}$ & $<0.001$ & $<0.001$ & $<0.001$ \\
df & \multicolumn{2}{|c}{} \\
p Value & \multicolumn{2}{|c|}{} \\
\hline
\end{tabular}

\section{References}

[1] Adolescents in India. A Profile. World Health Organization. 2003. http://www.whoindia.org/LinkFiles/Adolescent_Health_and_Development_(AHD)_UNFPA_Country_Report.pdf (Accessed on August 12, 2011).

[2] Banerjee I, Chakraborty S, Bhattacharyya NG, Bandyopadhyay S, Saiyed HN, Mukherjee D. A cohort study of correlation between body mass index and age at menarche in healthy Bengali girls. J Indian Med Assoc 2007; 105:75-8.

[3] Rao S, Joshi S, Kanade A. Height velocity, body fat and menarcheal age of Indian girls. Indian Pediatr 1998; 35:619-28.

[4] Singh MM, Devi R, Garg S, Mehra M. Effectiveness of syndromic approach in management of reproductive tract infections in women. Indian J Med Sci 2001; 55(4):209-14.

[5] Wasserheit JN. The significance and scope of reproductive tract infections among Third World women. Suppl Int J Gynecol Obstet 1989; 3:145-68.

[6] Suryakantha AH, Community Medicine with recent advances. Jaypee Brothers, New Delhi. $2^{\text {nd }}$ edition $2010 ; 680$.

[7] Khanna A, Goyal RS, Bhawsar R. Menstrual practices and reproductive problems: a study of adolescent girls in Rajasthan. J Health Manag 2005;7:91-107

[8] El-Gilany AH, Badawi K. Menstrual hygiene among adolescent schoolgirls in Mansoura, Egypt. Reprod Health Matters 2005; 13:14752.

[9] Deo DS, Ghattargi CH. Perceptions and practices regarding menstruation: a comparative study in urban and rural adolescent girls. Indian J Community Med 2005;30:33-4

[10] Drakshayani Devi K, Venkata Ramaiah P. A study on menstrual hygiene among rural adolescent girls; Indian J Med Sci 1994; 48:13943

[11] Menstrual Hygiene: How Hygienic is the Adolescent Girl? A Dasgupta, M Sarkar: Indian Journal of Community Medicine; April 2008; 33(2):78-80 